\title{
Soil pH buffering capacity and nitrogen availability following compost application in a tropical acid soil
}

\begin{abstract}
Tropical acid soils are highly weathered as they exist under tropical environment with high rainfall and temperature throughout the year, which affects nitrogen availability. Soil organic nitrogen is important in estimating soil nitrogen availability. The combined use of urea and compost in this study was carried out to decrease sole dependence on urea, buffer soil acidification, and reduce nitrogen losses through leaching. Thus, soil buffering capacity, incubation, and organic nitrogen fractionation studies were conducted to determine soil buffering capacity, availability of total nitrogen, organic fractions nitrogen, and inorganic nitrogen in soil after 90 days of incubation following compost. Soil $\mathrm{pH}$, buffering capacity, total nitrogen, organic nitrogen fractions, exchangeable ammonium, and available nitrate were higher in all treatments with compost and combined use of urea and compost. Total hydrolyzable nitrogen, ammonium-nitrogen, (ammonium + amino sugar)-nitrogen, amino sugar-nitrogen, and amino acid-nitrogen were higher in soils with urea and compost suggesting that decomposition of soil organic fractions nitrogen into inorganic nitrogen (ammonium and available nitrate was affected by the addition of urea and compost. Urea can be amended with compost to regulate availability nitrogen in soil for crop use.
\end{abstract}

